\title{
In-situ detection of hydrogen-induced phase transitions in individual palladium nanocrystals
}

Andrea Baldi, ${ }^{1,2 *}$ Tarun C. Narayan, ${ }^{1 *}$ Ai Leen Koh, ${ }^{3}$ Jennifer A. Dionne $e^{1,4 \dagger}$

*These authors contributed equally to this work.

†To whom correspondence should be addressed; E-mail: jdionne@ stanford.edu.

${ }^{1}$ Department of Materials Science and Engineering, Stanford University, 496 Lomita Mall, Stanford, CA 94305, USA

${ }^{2}$ FOM Institute DIFFER, Dutch Institute for Fundamental Energy Research, Edisonbaan 14, 3439 MN Nieuwegein, The Netherlands

${ }^{3}$ Stanford Nanocharacterization Laboratory, Stanford University, Stanford, CA 94305, USA

${ }^{4}$ Stanford Institute for Materials and Energy Sciences, SLAC National Accelerator Laboratory, Menlo Park, CA 94025, USA

Many energy and information storage processes rely on solute intercalation into a solid matrix. Compared to their bulk counterparts, nanostructured materials appear to exhibit faster charging and discharging kinetics, extended life cycles, and size-tunable thermodynamics. However, in ensemble studies of these materials, it is often difficult to discriminate between intrinsic size-dependent properties and effects due to sample size and shape dispersity. Here, we detect the phase transitions of individual palladium nanocrystals during hydrogen absorption and desorption, using in-situ electron energy-loss spectroscopy (EELS) in an environmental transmission electron microscope. In contrast to ensemble measurements, we find that palladium nanocrystals undergo sharp transitions between the $\alpha$ and $\beta$ phases, and that surface effects dictate the size dependence of the hydro- 


\section{gen absorption pressures. Our results provide a general framework for monitoring phase}

transitions in individual nanocrystals in a reactive environment and highlight the importance of single-particle approaches to the characterization of nanostructured materials.

The study of phase transitions involving solute intercalation in nanomaterials is essential for a wide range of applications such as hydrogen storage ${ }^{1-3}$, battery charging ${ }^{4-8}$, memory switching ${ }^{9,10}$, and nanoparticle synthesis ${ }^{11}$. Metal hydrides, and palladium hydride in particular, represent a convenient model system of solute intercalation, as they are characterised by fast hydrogenation and dehydrogenation kinetics at readily accessible transition temperatures and pressures. While initial reports of hydrogen absorption in Pd date back to $1866^{12}$, establishing the intrinsic, nanoscale size-dependent thermodynamic properties of this system has only just begun ${ }^{1,3,13-15}$. Recent studies of nanoscale systems have revealed significant thermodynamic deviations from the bulk, likely attributable to their increased surface area-to-volume ratio, modified defect chemistry and increased interaction with the substrate ${ }^{1}$. For example, ensemble measurements of $\sim 3 \mathrm{~nm}$ and $\sim 7 \mathrm{~nm}$ Pd nanoparticles have suggested a continuous transition from the dilute $\alpha$ phase of $\mathrm{PdH}_{x}$ to a hydrogen-rich, lattice-expanded $\beta$ phase ${ }^{15}$. Sloped isotherms have also been observed in ensemble luminescence studies of Pd nanocubes with sizes ranging between 14 and $65 \mathrm{~nm}$, which suggest that the $\alpha$-to- $\beta$ phase transition in nano-metal hydrides is mediated by thermal fluctuations ${ }^{3}$. Such studies provide striking contrast with the sharp $\alpha$-to- $\beta$ phase transitions observed in bulk palladium, and could have profound consequences for materials design in energy and information storage applications. Still, it remains uncertain if some of the trends observed result from intrinsic size effects or from particle dispersity inherent in the ensemble measurement.

Single particle measurements would resolve this ambiguity, but observing phase transitions in individual nanoparticles is extremely challenging ${ }^{10,16,17}$, particularly in reactive gaseous 
or liquid environments. One elegant approach to sense hydrogenation in an individual Pd nanoparticle relies on shifts in the surface plasmon resonance of a nearby gold nano-antenna ${ }^{18-20}$. Upon hydrogenation of $\mathrm{Pd}$, the gold plasmon resonance shifts, either due to changes in the Pd permittivity ${ }^{19,20}$, which modifies the local dielectric environment, or in the Pd volume ${ }^{21}$, which changes the Pd-Au interparticle separation. While some single particle measurements have resembled ensemble results ${ }^{18}$, others have indicated a sharper $\alpha$-to- $\beta$ phase transition and definitive hysteresis ${ }^{19}$, more akin to bulk palladium.

The inconclusive findings of single-particle measurements reflect the difficulty in probing nanomaterial phase transitions. Studies based on plasmonic nanoantennas only show small frequency shifts or line width broadenings upon hydrogenation of a nearby Pd nanoparticle. Many single particle experiments suffer from limited control over the particle size, shape and crystallinity: so far, the palladium nanostructures in all single particle studies are either evaporated onto a substrate or grown as thin shells on gold nanoparticles. Additionally, strains due to elastic interaction of the nanoparticle with the support can severely impact the system's thermodynamics. To date, direct measurement of hydrogen absorption and desorption in a single unconstrained Pd nanocrystal remains an outstanding challenge.

Here, we present the first experimental observation of hydrogen absorption and desorption in individual Pd nanocrystals, thus elucidating their hydrogenation and dehydrogenation thermodynamics. Rather than relying on the surface plasmon resonance of a nearby nanoantenna, we probe the bulk plasmon resonance of the Pd nanoparticle. Importantly, the bulk plasmon resonance is directly correlated to the complex permittivity of a material ${ }^{22}$, which in turn is strongly dependent on the hydrogen concentration in palladium ${ }^{23}$. To excite and detect the bulk plasmon resonance, we rely on EELS in an environmental (scanning) transmission electron 
microscope $(\mathrm{E}(\mathrm{S}) \mathrm{TEM})$. Upon hydrogen absorption, individual Pd nanocrystals exhibit large red shifts (exceeding $2 \mathrm{eV}$ ) in their bulk plasmon resonance ${ }^{24,25}$, and these shifts are reversible upon desorption. Our environmental (S)TEM-EELS approach allows correlation of individual particle spectra with particle size and crystallinity with high spatial and energy resolution.

\section{Single particle isotherms}

Palladium nanocrystals terminated by $\{100\}$ planes were colloidally synthesised using a previously reported method that yields nanocubes with edge lengths ranging from 10 to $35 \mathrm{~nm}^{26}$. Figure 1 shows an aberration-corrected transmission electron micrograph of a representative nanocrystal. Palladium nanocubes have been chosen due to their single crystalline nature which facilitates the interpretation of their hydrogen absorption properties, thanks to the absence of defects and grain boundaries ${ }^{27}$. Further, the synthesised nanocrystal size range allows us to address outstanding questions about the nature of phase transitions and surface effects in zerodimensional nanomaterials ${ }^{3,28}$. The nanocrystals were dispersed on a $\mathrm{SiO}_{2}$ TEM membrane, mounted on a liquid-nitrogen cooled TEM holder and imaged in an E(S)TEM (Figure 1a). A particle map was first constructed from a low-magnification STEM image, and a subset of 12 Pd nanocubes with varying sizes was identified and tracked during the hydrogen loading experiment (Figure 1c). Monochromated EEL spectra were acquired through the centre of each Pd nanocube, first at increasing (loading) and then at decreasing (unloading) $\mathrm{H}_{2}$ pressures, while keeping the sample at $246.0 \pm 0.5 \mathrm{~K}$. This temperature allowed the measurement of complete loading/unloading Pd isotherms within the pressure range allowed in the transmission electron microscope $\left(p\left(\mathrm{H}_{2}\right)<1000 \mathrm{~Pa}\right)$. The measured peaks, which correspond to the bulk plasmon resonance of the nanocubes ${ }^{29}$, were used to track the hydrogenation state of Pd; a large and reversible shift in bulk plasmon resonance marks the transition from a diluted $\alpha$ phase (peak at 7.7 
$\mathrm{eV}$ ), to a concentrated $\beta$ phase (peak at $5.6 \mathrm{eV})^{24,25}$. Further details on the nanocube synthesis, the TEM grid preparation, and the EEL spectra acquisition can be found in the Supplementary Information (SI 1).

Figure 2a shows the raw EEL spectra recorded on a representative $23 \mathrm{~nm}$ Pd nanocube in its initial state, after hydrogen absorption, and after hydrogen desorption. A shift of $\sim 2 \mathrm{eV}$ of the bulk resonance energy upon hydrogen absorption and desorption is clearly visible before any background correction. The initial bulk resonance is fully recovered upon decrease of $\mathrm{H}_{2}$ pressure, indicating that the observed shifts are due to the hydrogenation and dehydrogenation of the Pd nanocube and not to beam contamination or other spurious effects. EEL spectra measured on the $\mathrm{SiO}_{2}$ substrate next to the $\mathrm{Pd}$ nanocube at the same $\mathrm{H}_{2}$ pressure are shown as white lines for comparison, and are used as background references. We elected to perform the experiments using an accelerating voltage of $80 \mathrm{kV}$, since the increased inelastic scattering cross section at lower voltage gives rise to better EEL signals. Furthermore, at $80 \mathrm{kV}$, Cherenkov radiation effects from the $\mathrm{SiO}_{2}$ substrate are absent ${ }^{30}$, giving rise to a featureless background in the energy loss region where the $\mathrm{Pd}$ and $\mathrm{PdH}_{x}$ peaks occur.

The complete EEL spectrum evolution of the $23 \mathrm{~nm} \mathrm{Pd}$ nanocube upon $\mathrm{H}_{2}$ pressure increase and decrease is shown in Figure 2b. At the beginning of the loading experiment (top), the nanocube is in its $\alpha$ phase, characterised by a bulk resonance peak of $\sim 7.7 \mathrm{eV}$. The bulk EELS signal remains approximately constant until a pressure of $98 \mathrm{~Pa}$, where a sudden $\alpha$-to- $\beta$ transition occurs, as indicated by the abrupt $2 \mathrm{eV}$ red shift of the EELS peak to $\sim 5.7 \mathrm{eV}$. Importantly, the hydrogenation process is fully reversible, with the Pd nanocube returning to its $\alpha$ phase upon $\mathrm{H}_{2}$ pressure decrease.

From the EELS peak positions at varying pressures, we construct full loading and un- 
loading isotherms for 12 individual Pd nanocrystals with sizes ranging from $29 \mathrm{~nm}$ down to $13 \mathrm{~nm}$. These isotherms are plotted in Figure 3 and overlaid on the STEM images of the corresponding nanoparticles. As a function of hydrogen pressure and particle size, several trends can be noted. First, all particles larger than $15 \mathrm{~nm}$ show very abrupt transitions between the $\alpha$ and $\beta$ phases (top two rows). This result is in striking contrast to the sloped transition regions that characterise ensemble measurements of Pd nanocubes of similar sizes ${ }^{3}$, and suggests that ensemble results are influenced by the distribution in particle size, shape, and surface chemistry of the nanocubes. Second, particles smaller than $15 \mathrm{~nm}$ do exhibit sloped transition regions, suggesting that they have more complex loading thermodynamics. Third, the nanocube isotherms show large hysteresis gaps between their loading and unloading pressures. Fourth, the nanocubes exhibit size-dependent loading pressures, with all particles loading below the bulk loading pressure (white dashed lines). The larger nanoparticles investigated in our study tend to load at higher equilibrium pressures than the smaller ones. The desorption pressures, on the other hand, do not follow any obvious trend. This effect could be due to beam-induced heating of the nanoparticles during EELS acquisition, which would generally cause smaller particles with smaller heat capacities to unload prematurely. As explained in SI 2, this effect only affects the unloading isotherms, while the loading isotherms remain unperturbed.

\section{Coherent versus incoherent intercalation mechanism}

The presence of a large hysteresis gaps in metal-hydride loading and unloading isotherms has been the subject of investigation for many years ${ }^{13}$. Two models have generally been proposed to explain this effect. In one interpretation, incoherent precipitates of the $\beta$ phase nucleate and grow in the $\alpha$ phase. The presence of a large interfacial energy barrier prevents phase transition from occurring at thermodynamic equilibrium (where the chemical potentials $\mu$ of hydrogen in 
the gas and in the $\alpha$ and $\beta$ phases satisfy: $\frac{1}{2} \mu_{\mathrm{H}_{2}}=\mu_{\alpha}=\mu_{\beta}$ ) and results in the opening of a hysteresis gap. An alternative explanation considers the two coexisting phases to be coherently bound, characterized by a smoothly-varying lattice constant between the $\alpha$ and $\beta$ phases ${ }^{31}$. This coherency results in a macroscopic elastic energy barrier that is proportional to the volume of the sample ${ }^{28}$. Importantly, such an energy barrier prevents the coexistence of the two phases at thermodynamic equilibrium and gives rise to a hysteresis gap that depends on the elastic constants of the materials and on the lattice mismatch between $\alpha$ and $\beta$.

In order to discriminate between these two competing mechanisms, we consider the critical size of a coherent precipitate, which is determined by the balance between the strain energy stored in the precipitate and the energy required to form a dislocation ${ }^{32}$. This critical size is known to increase upon nanostructuring ${ }^{33}$. Indeed, although the maximum dimension of a coherent hydride inclusion in bulk Pd is expected to be on the order of $4 \mathrm{~nm}^{27}$, two-dimensional Pd thin films undergo coherent phase transitions up to a thickness of $22 \mathrm{~nm}^{33}$. A complete theory for the critical coherent size in zero-dimensional nanocrystals has not yet been developed, but it is reasonable to expect an even larger limit for nanoparticles than the one found in thin films. An indication that a coherent mechanism could be responsible for the behaviour observed in our nanocrystals comes from an analysis of the thermodynamic stability of a dislocation loop in a Pd nanoparticle. For analytical tractability, we consider a spherical Pd nanoparticle and assume that loading occurs first at the surface ${ }^{2,34}$. This loading scenario gives rise to a core shell structure in which the core is in the $\alpha$ phase and the shell in the $\beta$ phase. Our calculations show that a coherent, dislocation-free interface between the core and the shell is expected for Pd nanospheres with diameters as large as $30 \mathrm{~nm}^{35}$ (see SI 3). Given that all the particles we investigated are smaller than this critical size, the formation of incoherent interfaces is energetically unfavourable and thus unlikely to occur. Our analysis therefore suggests that the two 
phases are coherently bound and that the $\alpha$ and $\beta$ phases do not coexist in the core of a single crystalline nanocube. Interestingly, a similar suppression of phase coexistence upon nanostructuring has been observed in Li-insertion compounds that exhibit large volume expansions upon ion intercalation, such as $\mathrm{TiO}_{2}$ and $\mathrm{FePO}_{4}{ }^{36,37}$.

\section{Surface stress induces size-dependent thermodynamics}

As shown in Figure 3, the loading equilibrium pressures of our nanocubes generally decrease with decreasing particle dimensions. This trend, which has also been observed in ensemble experiments $^{3}$, can be explained by considering the effect of surface stress on the thermodynamics of hydrogen absorption in nanoscale hydrides, as first proposed by Weissmüller and Lemier $^{38,39}$. It is known that hydrogen atoms saturate sites at grain boundaries and at the surface of nanocrystalline $\mathrm{Pd}$ at $\mathrm{H}_{2}$ partial pressures well below the $\alpha$-to- $\beta$ phase transition ${ }^{13,39-41}$. The thickness of such a surface region has been estimated to be of the order of $1 \mathrm{~nm}^{39,41}$. The hydrogen concentration in these sites remains unchanged during the hydrogen absorption in the interior of the particle. Following the approach of Weissmüller and Lemier ${ }^{38}$, we therefore model our nanocrystals as composed of a $\mathrm{PdH}_{x}$ core of hydrogen concentration $x$, surrounded by a 1-nm-thick shell of $\mathrm{PdH}_{x_{\text {shell }}}$, characterised by a constant hydrogen concentration $x_{\text {shell }}$. At low $\mathrm{H}_{2}$ partial pressures, $x_{\text {shell }}>x$, and the lattice mismatch between the core and the shell results in a hydrostatic tensile pressure on the core, which modifies the chemical potential of hydrogen in the solid. In the limit of a perfectly coherent core/shell interface, the hydrogen chemical potential in a $\mathrm{Pd}$ nanoparticle, $\mu_{\mathrm{H}, \text { nano }}$, can therefore be expressed as the hydrogen chemical potential in bulk $\mathrm{Pd}, \mu_{\mathrm{H}, \text { bulk }}$, plus a term $\Delta \mu_{\mathrm{H}, \text { stress }}$ that takes into account the surface stress $^{39,42}$

$$
\mu_{\mathrm{H}, \text { nano }}=\mu_{\mathrm{H}, \text { bulk }}+\Delta \mu_{\mathrm{H}, \text { stress }}=\mu_{\mathrm{H}, \text { bulk }}-3 \varepsilon K \bar{V}_{\mathrm{H}}
$$


where $K$ is the bulk modulus of $\mathrm{Pd}, \bar{V}_{\mathrm{H}}$ is the partial molar volume of hydrogen in $\mathrm{Pd}$, and $\varepsilon$ is the linear strain in the core due to the presence of an excess hydrogen concentration in the shell.

In equation (1), the term $\mu_{\mathrm{H}, b u l k}$ is given by Brodowsky ${ }^{43}$ and contains the electronic contribution due to the increase in Fermi energy upon hydrogen absorption, the elastic $\mathrm{H}-\mathrm{H}$ pair interaction and the configurational entropy. In order to derive an analytical expression for the linear strain in the core, $\varepsilon$, we again approximate a nanocube of edge length $h$ as a spherical core/shell particle with total diameter $h$ and shell thickness $t$. It has in fact been shown that lattice-mismatched cubes and spheres embedded into a host matrix give rise to similar strains ${ }^{44}$. The strain can therefore be expressed as ${ }^{45,46}$

$$
\varepsilon=\frac{\bar{V}_{\mathrm{H}}\left(x_{\text {shell }}-x\right)}{3 \bar{V}_{\mathrm{Pd}}+\bar{V}_{\mathrm{H}}} \cdot \frac{2 \hat{d}\left(3+3 \hat{d}+\hat{d}^{2}\right)}{3(1+\hat{d})^{3}} \cdot \frac{1-2 \nu}{1-\nu}
$$

where $\bar{V}_{\mathrm{Pd}}$ is the molar volume of $\mathrm{Pd}, \hat{d}=2 t /(h-2 t)$, and $\nu$ is the Poisson's ratio of palladium, that is assumed to be constant with hydrogen concentration ${ }^{47}$. Substituting equation (2) into equation (1) shows that the stress due to the excess hydrogen concentration at the surface introduces particle size-dependence in the chemical potential of hydrogen in palladium. Figure $4 \mathrm{a}$ shows the concentration dependence of the chemical potential of hydrogen in bulk Pd, $\mu_{\mathrm{H}, \text { bulk }}$, and how it is modified due to the presence of the stress term, $\Delta \mu_{\mathrm{H}, \text { stress }}$, for a $15 \mathrm{~nm} \mathrm{Pd}$ nanoparticle. The dashed horizontal line marks the chemical potential at the spinodal concentration, $x^{*}$ satisfying $\partial \mu / \partial x=0$, that corresponds to the beginning of the $\alpha$-to- $\beta$ phase transition for systems in which coexistence is suppressed ${ }^{31}$. From this concentration we can determine the hydrogen loading pressure for different particle sizes using the equilibrium condition:

$$
\left.\mu_{\mathrm{H}, \text { nano }}\right|_{x=x^{*}}=\frac{1}{2} \mu_{\mathrm{H}_{2}}=\frac{1}{2}\left(\mu_{\mathrm{H}_{2}}^{0}+k T \ln \frac{p_{\mathrm{H}_{2}}}{p^{0}}\right)
$$

where $\mu_{\mathrm{H}_{2}}^{0}$ and $p^{0}$ are the standard chemical potential and pressure of $\mathrm{H}_{2}$ gas, respectively. 
Figure $4 \mathrm{~b}$ shows a fit of our experimental loading pressures as a function of particle size, using equation (3). We find that a constant shell concentration $x_{\text {shell }}=0.51$ well describes the observed size-dependence. This value, which has been determined assuming a shell thickness of $1 \mathrm{~nm}$, is in reasonable agreement with the 0.44 subsurface hydrogen concentration measured in single-sized palladium clusters ${ }^{13}$. A surface layer thicker than $1 \mathrm{~nm}$ might be expected for $\{100\}$-terminated cubes, as it has been observed that hydrogen on $\operatorname{Pd}(100)$ populates subsurface sites in a region up to $4 \mathrm{~nm}$ in depth ${ }^{48}$. Assuming a thicker surface layer in our model would therefore yield a smaller shell concentration (further details of the surface stress model are given in SI 4). Interestingly, the hydrostatic tensile pressure exerted on the core by the expanded shell can only arise if the core and the shell are coherent. The observed size-dependence therefore further supports the coherent model for the $\alpha$-to- $\beta$ phase transition.

\section{Conclusions}

In summary, we have reported the first direct measurement of hydrogen absorption and desorption in individual palladium nanocrystals, using in-situ environmental (S)TEM-EELS. Loading and unloading isotherms of single-crystalline $\mathrm{Pd}$ nanocubes are characterised by sharp $\alpha$-to- $\beta$ phase transitions and large hysteresis gaps. Surface stress due to excess hydrogen concentration at the surface of the nanocrystals can account for the size dependence of the equilibrium loading pressures. The loading and unloading isotherms of individual nanocrystals are consistent with a coherent loading process, in which phase coexistence is suppressed. Such an intercalation mechanism could be used to interpret a wide range of solid state reactions in which a reservoir of intercalants is in equilibrium with a coherent host lattice, such as Li-ion insertion in nanostructured electrodes ${ }^{5,7}$ and ion exchange reactions in quantum dots ${ }^{11,17}$. More generally, our in-situ detection scheme offers new avenues to study phase-transitions of single nanoparticles 
in a wide-range of reactive environments, with as-of-yet unknown thermodynamic properties.

\section{Methods}

Nanocube synthesis Single-crystalline $\{100\}$-terminated Pd nanocubes were synthesised following a previously reported method ${ }^{26}$. The particles were stabilised with hexadecyltrimethylammonium bromide (CTAB) and were washed twice with water to remove excess ligands. The synthesised nanoparticles had an average edge length of $25 \mathrm{~nm}$, based on about 500 particles measured by TEM.

In-situ environmental STEM EELS of individual Pd nanocubes. $10 \mu \mathrm{l}$ of the cleaned nanocubes were dispersed onto a 20-nm-thick $\mathrm{SiO}_{2}$ membrane (SPI Supplies) and allowed to dry in air for 40 minutes. Prior to TEM, the $\mathrm{SiO}_{2}$ membrane was plasma cleaned to remove organic contamination sources and to render the surface more hydrophilic. The sample was mounted onto a TEM cryo holder, that allowed sample temperature control to within $\pm 0.1 \mathrm{~K}$. All experiments were carried out using a FEI Titan 80-300 environmental (scanning) transmission electron microscope $(\mathrm{E}(\mathrm{S}) \mathrm{TEM})$ operated at $80 \mathrm{kV}$. The microscope was equipped with a monochromator, an aberration corrector in the image forming (objective) lens and a Gatan 966 Quantum electron energy loss (EEL) spectrometer. EELS experiments were performed in monochromated STEM mode using a $50 \mu \mathrm{m}$ C3 aperture, a $48 \mathrm{~mm}$ camera length, a $2.5 \mathrm{~mm}$ spectrometer entrance aperture and at a dispersion of $0.01 \mathrm{eV}$ per channel. These settings correspond to a convergence semi-angle of $8.7 \mathrm{mrad}$ and a collection semi-angle of $20.2 \mathrm{mrad}$. Before acquisition of the equilibrium isotherms, the Pd nanocubes were loaded and unloaded with hydrogen three times to relax stresses in the nanoparticles (see SI 1.4 for details). The sample was then cooled to $246 \pm 0.5 \mathrm{~K}$ and the $\mathrm{H}_{2}$ (99.9999\%, Praxair) pressure was varied between 4 and $600 \mathrm{~Pa}$ using a home-built gas manifold system operated by a mass flow controller. 
The $\mathrm{H}_{2}$ pressure in the microscope chamber was monitored using an Edwards Barocell 600 capacitance manometer, with a precision of $\pm 3 \%$. At each pressure point, EEL spectra were acquired for all Pd nanocubes. During EELS acquisitions the STEM probe was defocused to a diameter of $7 \mathrm{~nm}$, to minimize electron dose and beam-induced hydrogen desorption. When the $\mathrm{H}_{2}$ pressure was being varied, the electron beam was kept in a "parking" position at a sample region away from the particles being investigated, and the sample was allowed to equilibrate for at least 10 minutes before the next data acquisition. Further details of the experimental procedure are in SI 1.

\section{References and Notes}

1. Pundt, A. Hydrogen in nano-sized metals. Adv. Eng. Mater. 6, 11-21 (2004).

2. Bérubé, V., Radtke, G., Dresselhaus, M. \& Chen, G. Size effects on the hydrogen storage properties of nanostructured metal hydrides: A review. Int. J. Energy Res. 31, 637-663 (2007).

3. Bardhan, R. et al. Uncovering the intrinsic size dependence of hydriding phase transformations in nanocrystals. Nature Mater. 12, 905-912 (2013).

4. Aricò, A. S., Bruce, P., Scrosati, B., Tarascon, J.-M. \& van Schalkwijk, W. Nanostructured materials for advanced energy conversion and storage devices. Nature Mater. 4, 366-377 (2005).

5. Meethong, N., Huang, H.-Y. S., Speakman, S. A., Carter, W. C. \& Chiang, Y.-M. Strain accommodation during phase transformations in olivine-based cathodes as a materials selection criterion for high-power rechargeable batteries. Adv. Funct. Mater. 17, 1115-1123 (2007). 
6. Bruce, P. G., Scrosati, B. \& Tarascon, J.-M. Nanomaterials for rechargeable lithium batteries. Angew. Chem. Int. Ed. 47, 2930-2946 (2008).

7. Chueh, W. C. et al. Intercalation Pathway in Many-Particle $\mathrm{LiFePO}_{4}$ Electrode Revealed by Nanoscale State-of-Charge Mapping. Nano Lett. 13, 866-872 (2013).

8. Ebner, M., Marone, F., Stampanoni, M. \& Wood, V. Visualization and Quantification of Electrochemical and Mechanical Degradation in Li Ion Batteries. Science 342, 716-720 (2013).

9. Jeong, J. et al. Suppression of Metal-insulator Transition in $\mathrm{VO}_{2}$ by Electric Field-Induced Oxygen Vacancy Formation. Science 339, 1402-1405 (2013).

10. Ohno, T. et al. Short-term plasticity and long-term potentiation mimicked in single inorganic synapses. Nature Mater. 10, 591-595 (2011).

11. D. H. Son, Y. Y., S. M. Hughes \& Alivisatos, A. P. Cation Exchange Reactions in Ionic Nanocrystals. Science 306, 1009-1012 (2004).

12. Graham, T. On the Absorption and Dialytic Separation of Gases by Colloid Septa. Philos. Trans. Roy. Soc. London 156, 399 (1866).

13. Sachs, C. et al. Solubility of hydrogen in single-sized palladium clusters. Phys. Rev. B 64, 075408 (2001).

14. Pundt, A. \& Kirchheim, R. Hydrogen in metals: Microstructural aspects. Annu. Rev. Mater. Res. 36, 555-608 (2006).

15. Yamauchi, M., Ikeda, R., Kitagawa, H. \& Takata, M. Nanosize effects on hydrogen storage in palladium. J. Phys. Chem. C 112, 3294-3299 (2008). 
16. Zheng, H. et al. Observation of Transient Structural-Transformation Dynamics in a $\mathrm{Cu}_{2} \mathrm{~S}$ Nanorod. Science 333, 206-209 (2011).

17. Routzahn, A. L. \& Jain, P. K. Single-Nanocrystal Reaction Trajectories Reveal Sharp Cooperative Transitions. Nano Lett. 12, 987-992 (2014).

18. Liu, N., Tang, M. L., Hentschel, M., Giessen, H. \& Alivisatos, A. P. Nanoantenna-enhanced gas sensing in a single tailored nanofocus. Nature Mater. 10, 631-636 (2011).

19. Shegai, T. \& Langhammer, C. Hydride formation in single palladium and magnesium nanoparticles studied by nanoplasmonic dark-field scattering spectroscopy. Adv. Mater. 23, 4409-4414 (2011).

20. Tang, M. L., Liu, N., Dionne, J. A. \& Alivisatos, A. P. Observations of shape-dependent hydrogen uptake trajectories from single nanocrystals. J. Am. Chem. Soc. 133, 1322013223 (2011).

21. Tittl, A., Kremers, C., Dorfmüller, J., Chigrin, D. N. \& Giessen, H. Spectral shifts in optical nanoantenna-enhanced hydrogen sensors. Opt. Mater. Express 2, 111-118 (2012).

22. García de Abajo, F. J. Optical excitations in electron microscopy. Rev. Mod. Phys. 82, 209-275 (2010).

23. Gremaud, R., Slaman, M., Schreuders, H., Dam, B. \& Griessen, R. An optical method to determine the thermodynamics of hydrogen absorption and desorption in metals. Appl. Phys. Lett. 91, 231916 (2007).

24. Bennett, P. A. \& Fuggle, J. C. Electronic structure and surface kinetics of palladium hydride studied with x-ray photoelectron spectroscopy and electron-energy-loss spectroscopy. Phys. Rev. B 26, 6030-6039 (1982). 
25. Liu, D. R. \& Brown, L. M. Characterization of palladium hydride films by electron energy loss spectroscopy and electron diffraction. Acta Metall. 36, 2597-2604 (1988).

26. Niu, W. et al. Seed-mediated growth of nearly monodisperse palladium nanocubes with controllable sizes. Cryst. Growth Des. 8, 4440-4444 (2008).

27. Pundt, A. et al. Hydrogen sorption in elastically soft stabilized Pd-clusters. J. Alloys. Compd. 293-295, 480-483 (1999).

28. Schwarz, R. B. \& Khachaturyan, A. G. Thermodynamics of open two-phase systems with coherent interfaces: Application to metal-hydrogen systems. Acta Mater. 54, 313-323 (2006).

29. Scholl, J. A., Koh, A. L. \& Dionne, J. A. Quantum plasmon resonances of individual metallic nanoparticles. Nature 483, 421-427 (2012).

30. Jung, H. J., asnd P. B. Van Stockum, N. P. D., Koh, A. L., Sinclair, R. \& Prinz, F. B. Spatial variation of available electronic excitations within individual quantum dots. Nano Lett. 13, 716-721 (2013).

31. Wagner, H. Topics in Applied Physics: Hydrogen in Metals I (Springer, Berlin, 1978). Edited by G. Alefeld and J. Völkl.

32. Nörthemann, K. \& Pundt, A. Coherent-to-semi-coherent transition of precipitates in niobium-hydrogen thin lms. Phys. Rev. B 78, 014105 (2008).

33. Wagner, S. et al. Achieving coherent phase transition in palladium-hydrogen thin films. Scripta Mater. 64, 978-981 (2011).

34. Langhammer, C., Zhdanov, V. P., Zorić, I. \& Kasemo, B. Size-Dependent Kinetics of Hydriding and Dehydriding of Pd Nanoparticles. Phys. Rev. Lett. 104, 135502 (2010). 
35. Nix, W. D. (2013). Personal communication.

36. Wagemaker, M., Borghols, W. J. H. \& Mulder, F. M. Large Impact of Particle Size on Insertion Reactions. A Case for Anatase $\mathrm{Li}_{x} \mathrm{TiO}_{2}$. J. Am. Chem. Soc. 129, 4323-4327 (2007).

37. Liu, H. et al. Capturing metastable structures during high-rate cycling of $\mathrm{LiFePO}_{4}$ nanoparticle electrodes. Science 344, 1252817 (2014).

38. Weissmüller, J. \& Lemier, C. On the size dependence of the critical point of nanoscale interstitial solid solutions. Phil. Mag. Lett. 80, 411-418 (2000).

39. Lemier, C. \& Weissmüller, J. Grain boundary segregation, stress and stretch: Effects on hydrogen absorption in nanocrystalline palladium. Acta Mater. 55, 1241-1254 (2007).

40. Mütschele, T. \& Kirchheim, R. Segregation and diffusion of hydrogen in grain boundaries of palladium. Scripta Metall. 21, 135-140 (1987).

41. Mütschele, T. \& Kirchheim, R. Hydrogen as a probe for the average thickness of a grain boundary. Scripta Metall. 21, 1101-1104 (1987).

42. Griessen, R. \& Feenstra, R. Volume changes during hydrogen absorption in metals. $J$. Phys. F: Met. Phys. 15, 1013-1019 (1985).

43. Brodowsky, H. On the non-ideal solution behavior of hydrogen in metals. Ber. Bunsenges. Phys. Chem. 76, 740-746 (1972).

44. Andreev, A. D., Downes, J. R., Faux, D. A. \& O’Reilly, E. P. Strain distributions in quantum dots of arbitrary shape. J. Appl. Phys. 86, 297-305 (1999). 
45. Rockenberger, J. et al. The contribution of particle core and surface to strain, disorder and vibrations in thiolcapped CdTe nanocrystals. J. Chem. Phys. 108, 7807-7815 (1998).

46. Fahmy, A. A. \& Ragay, A. N. Thermal-Expansion Behavior of Two-Phase Solids. J. Appl. Phys. 41, 5108-5111 (1970).

47. Hsu, D. K. \& Leisure, R. G. Elastic constants of palladium and $\beta$-phase palladium hydride between 4 and 300 K. Phys. Rev. B 20, 1339-1344 (1979).

48. Wilde, M., Matsumoto, M., Fukutani, K. \& Aruga, T. Depth-resolved analysis of subsurface hydrogen absorbed by Pd(100). Surf. Sci. 482-485, 346-352 (2001).

49. Clewley, J. D., Curran, T., Flanagan, T. B. \& Oates, W. A. Thermodynamic Properties of Hydrogen and Deuterium Dissolved in Palladium at Low Concentrations over a Wide Temperature Range. J. Chem. Soc. Faraday Trans. 1 69, 449-458 (1972).

50. Wicke, E. \& Brodowsky, H. Topics in Applied Physics: Hydrogen in Metals II (Springer, Berlin, 1978). Edited by G. Alefeld and J. Völkl.

Acknowledgements We gratefully acknowledge scientific feedback and discussions with Jonathan Scholl, Prof. William D. Nix, Prof. Ronald Griessen, and Prof. Astrid Pundt. JAD acknowledges support from a Stanford Terman Fellowship, a Hellman Fellowship, an Air Force Office of Scientific Research Young Investigator Grant (FA9550-11-1-0024) and a National Science Foundation CAREER Award (DMR-1151231). This work was supported in part by a SLAC National Accelerator Laboratory LDRD award in concert with the Department of Energy, Office of Basic Energy Sciences, Division of Materials Sciences and Engineering, under contract DEAC02-76SF00515. Work was also supported 
by the research program Fellowships for Young Energy Scientists (YES!) of the Foundation for Fundamental Research on Matter (FOM), which is financially supported by the Netherlands Organisation for Scientific Research (NWO), and by an award from the Department of Energy (DOE) Office of Science Graduate Fellowship Program administered by the Oak Ridge Institute for Science and Education for the DOE. ORISE is managed by Oak Ridge Associated Universities (ORAU) under DOE contract number DE-AC05-06OR23100. All opinions expressed in this paper are the authors and do not necessarily reflect the policies and views of NSF, DOE, ORAU, or ORISE.

Author contributions A.B., T.C.N. and J.A.D. designed the experiments and A.B., T.C.N. and AL.K. performed the experiments. A.B. and T.C.N. analysed the data and wrote the initial draft of the manuscript. J. D. supervised the project, and all authors discussed the results and contributed to final manuscript preparation.

Competing financial interests The authors declare that they have no competing financial interests.

Correspondence Correspondence and requests for materials should be addressed to J.A.D. (email: jdionne@stanford.edu). 

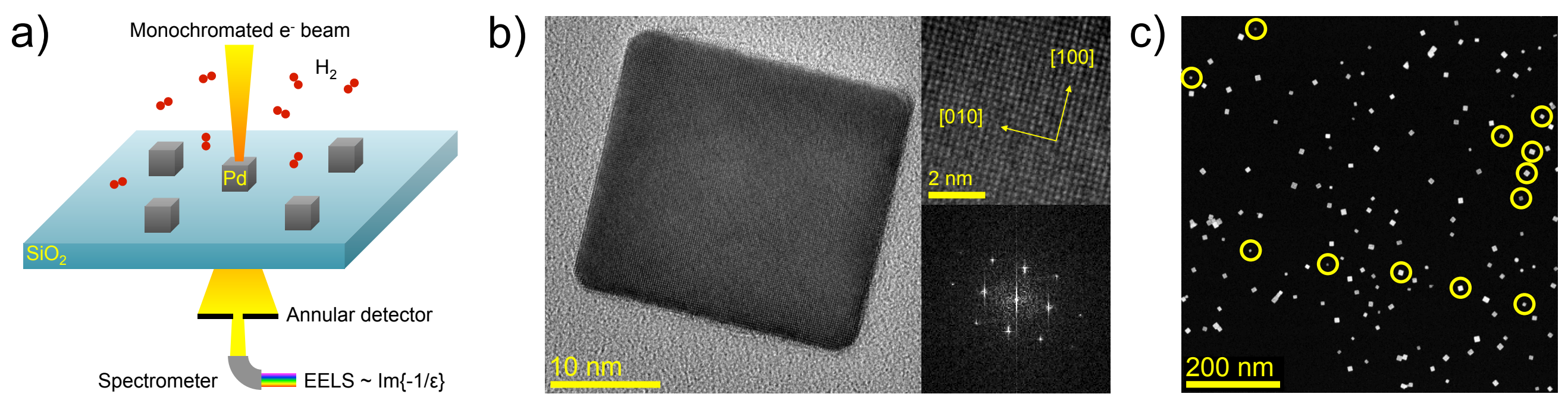

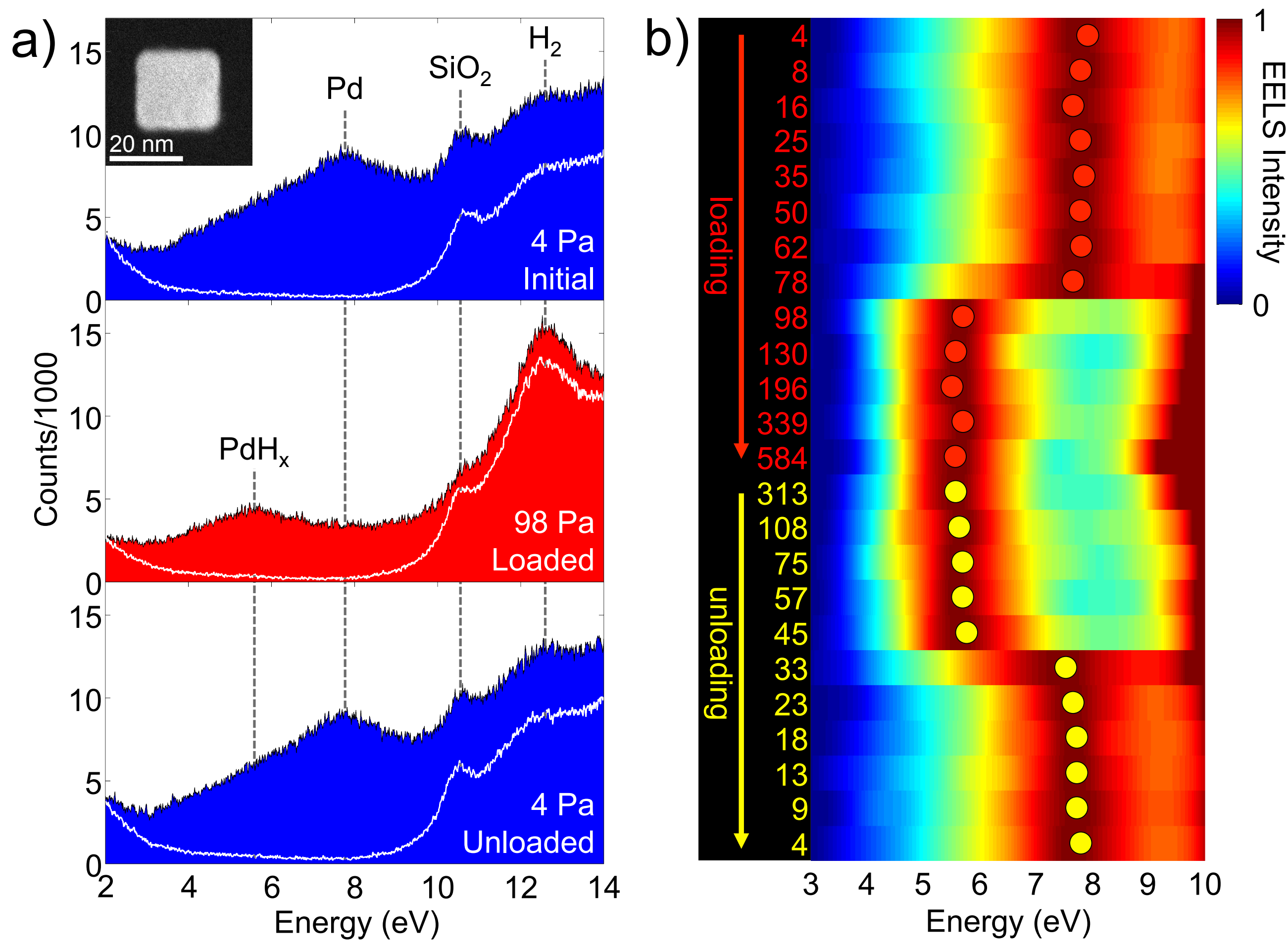


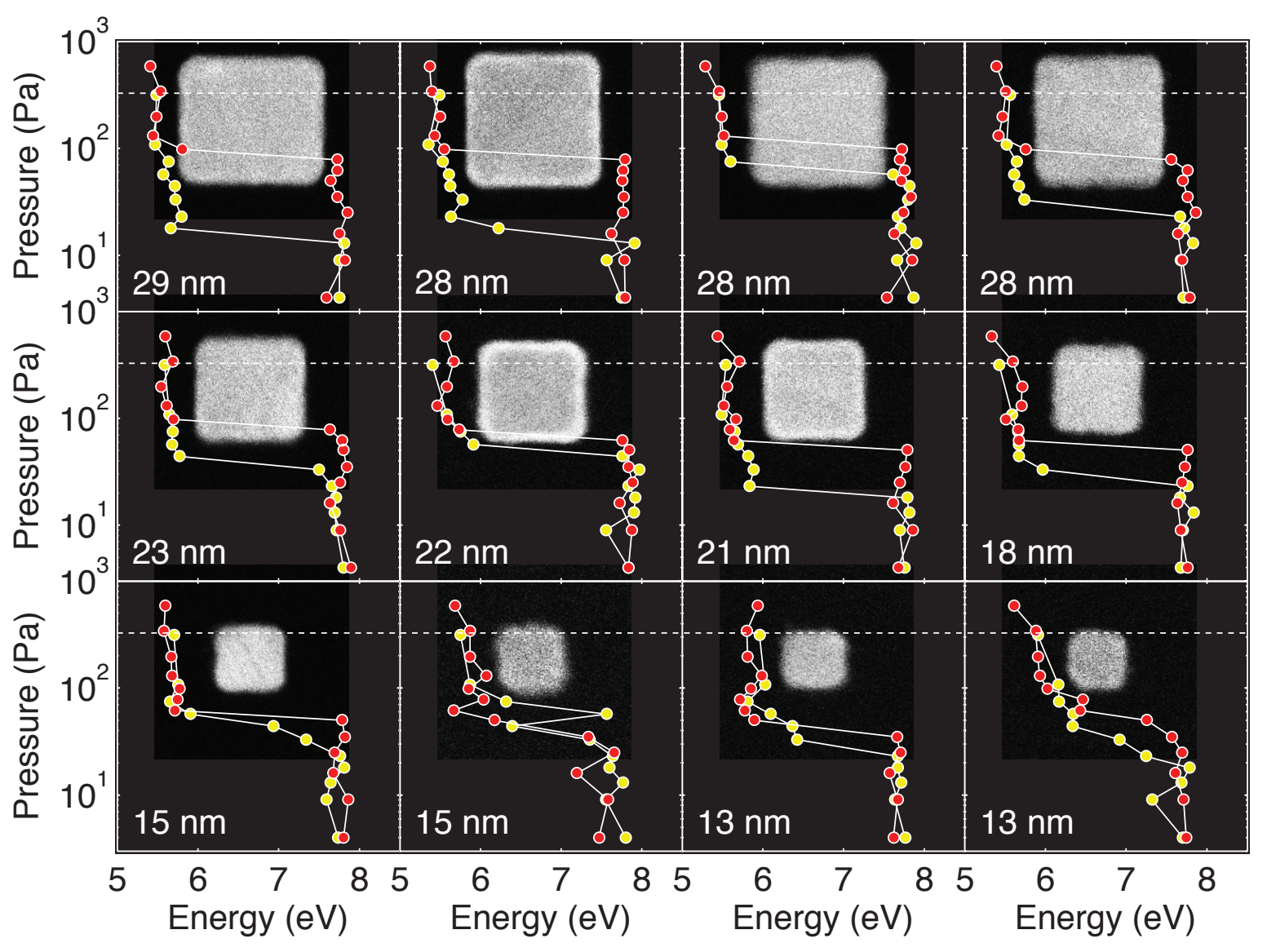


Figure 1 Pd nanocube TEM and In-situ EELS setup. a) Schematic of the in-situ measurement setup. EELS spectra are collected by focusing a monochromated $80 \mathrm{kV}$ electron beam in the centre of a Pd nanocube exposed to a controlled $\mathrm{H}_{2}$ pressure. b) Aberration-corrected TEM image of a representative single-crystalline Pd nanocube on an ultra thin $(\sim 3 \mathrm{~nm})$ carbon substrate. The adjacent panels show a zoomed-in view of the cube and its corresponding Fourier transform. c) Low magnification annular darkfield STEM image showing the $12 \mathrm{Pd}$ nanocubes characterised in this study (yellow circles). The nanocubes are dispersed on a $20 \mathrm{~nm}$ thick $\mathrm{SiO}_{2} \mathrm{TEM}$ membrane. All the measured particles are separated from each other by at least $2 x$ their edge length to minimize any inter-particle near-field interaction.

Figure 2 EEL spectra of a single Pd nanocrystal at varying $\mathbf{H}_{2}$ pressures. a) Filled areas: raw EEL spectra recorded on the $23 \mathrm{~nm} \mathrm{Pd}$ nanocube shown in the inset at the beginning of the experiment (top panel, $p\left(\mathrm{H}_{2}\right)=4 \mathrm{~Pa}$ ), after hydrogen absorption (centre panel, $p\left(\mathrm{H}_{2}\right)=98 \mathrm{~Pa}$ ), and after hydrogen desorption (bottom panel, $p\left(\mathrm{H}_{2}\right)=4 \mathrm{~Pa}$ ). The peaks at energy losses of $10.6 \mathrm{eV}$ and $12.6 \mathrm{eV}$ originate from the $\mathrm{SiO}_{2}$ substrate and the $\mathrm{H}_{2}$ gas, respectively. White lines: background EEL spectra recorded on a $\mathrm{SiO}_{2}$ area close to the $\mathrm{Pd}$ nanocube (the spectra have been rescaled for clarity). d) Complete EEL spectrum evolution upon $\mathrm{H}_{2}$ pressure increase (loading) and decrease (unloading). The corresponding $\mathrm{H}_{2}$ pressures in $\mathrm{Pa}$ are indicated next to each spectrum. The spectra are smoothed with a locally weighted $2^{\text {nd }}$ order polynomial (LOESS) regression and normalised by the height of the bulk plasmon resonance peak. The peak maxima are indicated with a red (loading) or yellow (unloading) circle. The increased signal at $E>9 \mathrm{eV}$ at high $\mathrm{H}_{2}$ pressures is due to increased electron energy losses in the $\mathrm{H}_{2}$ gas. 
Figure 3 Single particle isotherms. Loading (red) and unloading (yellow) isotherms and corresponding STEM images (shown to scale) of the palladium nanocubes characterised in this study. All isotherms are measured at $246 \mathrm{~K}$. The dashed white lines at $338 \mathrm{~Pa}$ correspond to the bulk loading pressure of palladium at $246 \mathrm{~K}$, obtained from Brodowsky ${ }^{43}$, and using the standard partial molar enthalpy and entropy given by Clewley $^{49}$ (see also SI 4). The error bars for both pressure and energy are within the size of the data points.

Figure 4 Surface stress effect on the loading equilibrium pressures. a) Concentration dependence of the chemical potential of hydrogen in bulk $\mathrm{Pd}, \mu_{\mathrm{H}, \text { bulk }}$ (blue solid line), and in a Pd nanoparticle with a $15 \mathrm{~nm}$ diameter, $\mu_{\mathrm{H}, \text { nano }}$ (magenta solid line). The difference between the two is the stress-induced term $\Delta \mu_{\mathrm{H}, \text { stress }}$ (red solid line), which accounts for the tensile hydrostatic pressure exerted on the core by the hydrogensaturated $1 \mathrm{~nm}$ thick shell. The concentration in the shell is fixed at $x_{\text {shell }}=0.51$, which corresponds to the value fitted to our experimental loading pressures. The plots for $\mu_{\mathrm{H}, \text { bulk }}$ and $\mu_{\mathrm{H}, \text { nano }}$ have been shifted vertically by $+0.1 \mathrm{eV}$ for easier comparison with $\Delta \mu_{\mathrm{H}, \text { stress. }}$. b) Equilibrium loading pressures measured on $12 \mathrm{Pd}$ nanocubes as a function of the nanocube average edge length, $h$, calculated as the square-root of the particle area in the STEM images. The vertical uncertainties span the difference in pressure between the highest pressure in the $\alpha$ phase and the lowest pressure in the $\beta$ phase. The dashed lines indicate the calculated loading pressures using equation (3), with $x_{\text {shell }}=0.41, x_{\text {shell }}=0.51$ (the fitted value), and $x_{\text {shell }}=0.61$. In the fit we used $t=1 \mathrm{~nm}^{39,41}, \bar{V}_{\mathrm{H}}=2.61 \AA^{3} /$ atom $=1.57 \cdot 10^{-6} \mathrm{~m}^{3} / \mathrm{mol}^{50}, \bar{V}_{\mathrm{Pd}}=8.85 \cdot 10^{-6}$ $\mathrm{m}^{3} / \mathrm{mol}, K=187 \mathrm{GPa}^{39}, \nu=0.39^{39}$, and $T=246 \mathrm{~K}$. The inset shows a schematic of the core/shell spherical model we used to approximate our Pd nanocubes. 